\title{
4 Dispersion Representations
}

\subsection{From Cauchy Integral Representation to Hilbert Transforms}

A recurrent theme thus far is that an analytic function is completely determined by its singularities. We have seen this principle manifested successively for constants (Liouville's Theorem), rational functions and most recently, meromorphic functions with simple poles. In this Section we shall take another step by discovering how functions with branch points can be represented in terms of their behaviour along the associated branch cuts. In so doing, we shall encounter a mathematical tool that has been found to be very useful in several fields of modern physics.

Suppose that we have a function $f(z)$ which is holomorphic throughout the complex plane except for a cut extending from $x_{0}$ to $\infty$ along the positive real axis. Suppose further that $f(z)$ satisfies the conditions

$$
f\left(z^{\star}\right)=f^{\star}(z),|f(z)| \rightarrow 0 \text { as }|z| \rightarrow \infty \text {, and }\left|\left(z-x_{0}\right) f(z)\right| \rightarrow 0 \text { as }\left|z-x_{0}\right| \rightarrow 0 .
$$

The first of these conditions is sometimes referred to as a reality condition since, as we shall see in Section 4.2, it implies that $f(x)$ is real for $x<x_{0}$.

The Cauchy Integral representation of $f(z)$ at a point $z$ not on the cut is

$$
f(z)=\frac{1}{2 \pi i} \int_{C} \frac{f(\zeta)}{\zeta-z} d \zeta
$$

where $C$ is a contour like that shown in Figure 4.1. The contribution to the integral from the large circle in $C$ goes to zero as the circle's radius $R \rightarrow \infty$. Moreover, the contribution from the small circle about $z=x_{0}$ vanishes as $\varepsilon \rightarrow 0$. Thus, in this double limit we have

$$
\begin{aligned}
f(z) & =\lim _{\varepsilon \rightarrow 0} \frac{1}{2 \pi i}\left\{\int_{x_{0}+i \varepsilon}^{\infty+i \varepsilon} \frac{f(\zeta)}{\zeta-z} d \zeta-\int_{x_{0}-i \varepsilon}^{\infty-i \varepsilon} \frac{f(\zeta)}{\zeta-z} d \zeta\right\} \\
& =\lim _{\varepsilon \rightarrow 0} \frac{1}{2 \pi i}\left\{\int_{x_{0}}^{\infty} \frac{f(\xi+i \varepsilon)}{\xi-z+i \varepsilon} d \xi-\int_{x_{0}}^{\infty} \frac{f(\xi-i \varepsilon)}{\xi-z-i \varepsilon} d \xi\right\} .
\end{aligned}
$$

Since $z$ is not on the cut, we can neglect the $\pm i \varepsilon$ in the denominators of the last two integrals and write

$$
f(z)=\frac{1}{2 \pi i} \int_{x_{0}}^{\infty} \lim _{\varepsilon \rightarrow 0} \frac{[f(\xi+i \varepsilon)-f(\xi-i \varepsilon)]}{\xi-z} d \xi
$$

The numerator of the integrand in (4.1.2) is the discontinuity of $f(z)$ across the cut. Thus, all we need in order to compute $f(z)$ at any point where it is holomorphic 


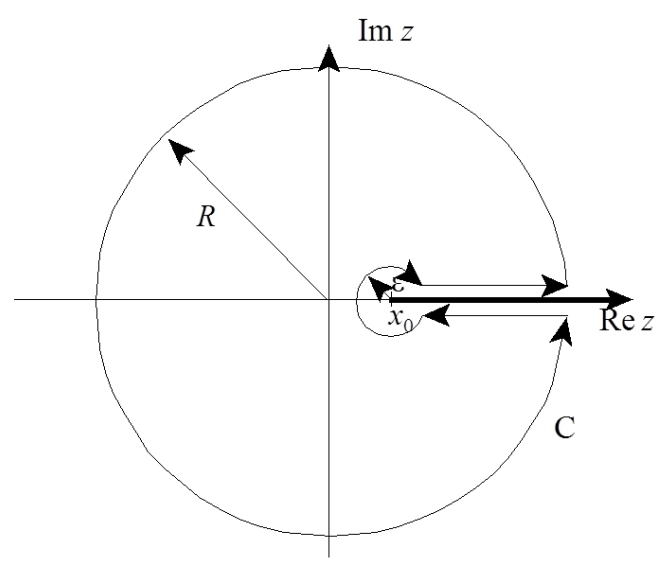

Figure 4.1: The contour used to derive a dispersion representation for a function with a cut along the real; axis segment $x_{0} \leq x<\infty$.

is a knowledge of its behaviour along its branch cut singularity. One of our initial assumptions allows us to cast this relationship in a somewhat simpler form. Since $f^{\star}(z)=f\left(z^{\star}\right)$, we have

$$
\begin{aligned}
\lim _{\varepsilon \rightarrow 0}[f(x+i \varepsilon)-f(x-i \varepsilon)] & =\lim _{\varepsilon \rightarrow 0}\left[f(x+i \varepsilon)-f^{\star}(x+i \varepsilon)\right] \\
& =\lim _{\varepsilon \rightarrow 0} 2 i \operatorname{Im} f(x+i \varepsilon)=2 i \operatorname{Im} f_{+}(x)
\end{aligned}
$$

where we have defined

$$
f_{+}(x)=\lim _{\delta \rightarrow 0^{+}} f(x+i \delta) .
$$

Inserting (4.1.3) into (4.1.2) gives us

$$
f(z)=\frac{1}{\pi} \int_{x_{0}}^{\infty} \frac{\operatorname{Im} f_{+}(\xi)}{\xi-z} d \xi .
$$

This is an example of what physicists call a dispersion relation, a title which has its origin in the theory of optical and X-ray dispersion at the turn of the $\left(19^{\text {th }}\right)$ century. In optics a dispersion relation is an integral relationship between the refractive (real) and absorptive (imaginary) parts of the refractive index, the variable of integration being the frequency of the incident radiation. Whether mindful of the historic and scientific significance of the first such relationship, derived by Kramers and Kronig, or appreciative of the virtues of a spare vocabulary, physicists apply the term "dispersion relation" to any integral equation that links the real and imaginary parts of an analytic function.

For reasons which we will explore at a later date the classical dispersion relations were derived for functions for which one can only assume holomorphy in the upper 


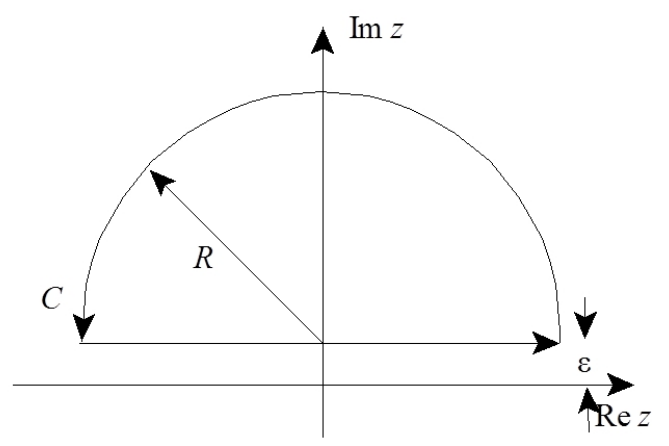

Figure 4.2: The contour used to derive the dispersion relation for a function $f(z)$ that is holomorphic only for $\operatorname{Im} z>0$.

half-plane, $\operatorname{Im} z>0$. Thus, in place of the contour of Figure 4.1, we must use a semicircle like that shown in Figure 4.2. Again using Cauchy's Integral Representation as our starting point and taking the double limit as $R \rightarrow \infty$ and $\varepsilon \rightarrow 0$, we obtain in place of (4.1.2)

$$
f(z)=\lim _{\varepsilon \rightarrow 0} \frac{1}{2 \pi i} \int_{-\infty}^{\infty} \frac{f(\xi+i \varepsilon)}{\xi-z+i \varepsilon} d \xi=\frac{1}{2 \pi i} \int_{-\infty}^{\infty} \frac{f_{+}(\xi)}{\xi-z} d \xi
$$

We now let $z$ approach the real axis from above so that (4.1.6) becomes

$$
f_{+}(x)=\lim _{\delta \rightarrow 0^{+}} \frac{1}{2 \pi i} \int_{-\infty}^{\infty} \frac{f_{+}(\xi)}{\xi-x+i \delta} d \xi .
$$

But, using equation (3.3.36), this can be rewritten in terms of a principal value integral:

$$
f_{+}(x)=\frac{1}{2 \pi i} \wp \int_{-\infty}^{\infty} \frac{f_{+}(\xi)}{\xi-\chi} d \xi+\frac{1}{2} f_{+}(x)
$$

or,

$$
f_{+}(x)=\frac{1}{\pi i} \wp \int_{-\infty}^{\infty} \frac{f_{+}(\xi)}{\xi-x} d \xi
$$

Finally, we take the real and imaginary parts of this equation to obtain the classical dispersion relations

$$
\operatorname{Re} f_{+}(x)=\frac{\wp}{\pi} \int_{-\infty}^{\infty} \frac{\operatorname{Im} f_{+}(\xi)}{\xi-x} d \xi
$$




$$
\operatorname{Im} f_{+}(x)=\frac{-\wp}{\pi} \int_{-\infty}^{\infty} \frac{\operatorname{Re} f_{+}(\xi)}{\xi-x} d \xi .
$$

From the point of view of a mathematician these relations establish that the real and imaginary parts of $f_{+}(x)$ are reciprocal Hilbert transforms.

David Hilbert (1862-1943) was a German mathematician who laid the foundations for much of modern mathematics including algebraic geometry and algebraic number theory. Over his lifetime, he made important contributions to the axiomatic foundations of geometry, integral equations, the calculus of variations, theoretical physics (which he thought was too difficult to be the domain of physicists alone), and mathematical logic.

In most applications $\operatorname{Im} f_{+}(x)$ is known from experiment and $\operatorname{Re} f_{+}(x)$ is then determined by application of (4.1.8). However, since $x$ is typically a frequency or energy variable, measurements can only be made for values of $x \geq 0$. In that event a crossing symmetry such as $f_{+}(-x)=f_{+}^{\star}(x)$ has to be invoked to allow (4.1.8) and (4.1.9) to be written

$$
\operatorname{Re} f_{+}(x)=\frac{2}{\pi} \wp \int_{0}^{\infty} \frac{\xi \operatorname{Im} f_{+}(\xi)}{\xi^{2}-x^{2}} d \xi
$$

and

$$
\operatorname{Im} f_{+}(x)=\frac{-2 x}{\pi} \wp \int_{0}^{\infty} \frac{\operatorname{Re} f_{+}(\xi)}{\xi^{2}-x^{2}} d \xi
$$

respectively, which should look familiar to readers who have been introduced to the Kramers-Kronig relation for indices of refraction.

\subsection{Adding Poles and Subtractions}

Returning to functions whose analytic properties are known throughout the complex plane, we shall generalize the dispersion representation of equation (4.1.5) to accommodate functions possessing poles as well as cuts. Suppose that we have a function $f(z)$ which, in addition to the properties that resulted in (4.1.5), has simple poles at the points $z=z_{k}, k=1,2, \ldots, n$, none of which lie on the branch cut. Denoting the residue of $f(z)$ at $z=z_{k}$ by $r_{k}$, equation (4.1.2) now becomes

$$
f(z)+\sum_{k=1}^{n} \frac{r_{k}}{z_{k}-z}=\lim _{\varepsilon \rightarrow 0} \frac{1}{2 \pi i}\left\{\int_{x_{0}+i \varepsilon}^{\infty+i \varepsilon} \frac{f(\zeta)}{\zeta-z} d \zeta-\int_{x_{0}-i \varepsilon}^{\infty-i \varepsilon} \frac{f(\zeta)}{\zeta-z} d \zeta\right\} .
$$

Thus, (4.1.5) is replaced by

$$
f(z)=\sum_{k=1}^{n} \frac{r_{k}}{z-z_{k}}+\frac{1}{\pi} \int_{x_{0}}^{\infty} \frac{\operatorname{Im} f_{+}(\xi)}{\xi-z} d \xi .
$$


Notice that the sum in this equation is that of the principal parts of $f(z)$ at the poles $z=z_{k}$. One can show that this continues to be the case should any of the poles be of higher order than one.

A further generalization of (4.1.5) we should consider obtains when $f(z)$ does not vanish but is polynomial bounded as $|z| \rightarrow \infty$. If the only constraint at infinity is that $\left|f(z) / z^{n}\right| \rightarrow 0$ for some $n>0$, there is a variety of possible dispersion representations for $f(z)$ depending on what other information we have about the function. For example, if we know the value of $f(z)$ and its first $(n-1)$ derivatives at some point $z=z_{0}$, we can determine a dispersion representation for $f(z) /\left(z-z_{0}\right)^{n}$ and from it deduce a representation for $f(z)$ itself. Should we lack such detailed information about $f(z)$ at a specific point but at least possess knowledge of its value at each of a set of $n$ points, $z_{1}, \ldots, z_{n}$, we can invoke (4.2.1) to obtain a dispersion representation for $\frac{f(z)}{\left(z-z_{1}\right) \ldots\left(z-z_{n}\right)}$ and hence, one for $f(z)$ as well.

To illustrate what is involved, let us assume that $f(z)$ possesses all the properties that led us to the representation in (4.1.5) save one; instead of $|f(z)| \rightarrow 0$ as $|z| \rightarrow \infty$, we have $|f(z)| \rightarrow$ a non-zero constant. To compensate, we add one further piece of information about $f(z)$ : its value at the point $z=0$. We now have sufficient information to apply (4.2.1) to the function $f(z) / z$ which has a single simple pole at $z=0$ with residue $f(0)$. Thus, we obtain

$$
\frac{f(z)}{z}=\frac{f(0)}{z}+\frac{1}{\pi} \int_{x_{0}}^{\infty} \frac{\operatorname{Im} f_{+}(\xi)}{\xi(\xi-z)} d \xi
$$

or,

$$
f(z)=f(0)+\frac{z}{\pi} \int_{x_{0}}^{\infty} \frac{\operatorname{Im} f_{+}(\xi)}{\xi(\xi-z)} d \xi .
$$

This is called a subtracted dispersion relation because it can be obtained (notionally) by assuming the unsubtracted dispersion relation (4.1.5),

$$
f(z)=\frac{1}{\pi} \int_{x_{0}}^{\infty} \frac{\operatorname{Im} f_{+}(\xi)}{\xi-z} d \xi,
$$

subtracting from it its value at $z=0$,

$$
f(0)=\frac{1}{\pi} \int_{x_{0}}^{\infty} \frac{\operatorname{Im} f_{+}(\xi)}{\xi} d \xi,
$$

and then combining the two integrals by means of

$$
\frac{1}{\xi-z}-\frac{1}{\xi}=\frac{z}{\xi(\xi-z)} .
$$


Subtracted dispersion relations are often used even when the asymptotic behaviour of the function of interest does not make them mandatory. This is because the integral in (4.2.2) is less sensitive to high values of $\xi$ than is its counterpart in (4.1.5). Thus, the error committed in omitting experimentally inaccessible values of $\operatorname{Im} f_{+}(\xi)$ at high $\xi$ is decreased.

\subsection{Mathematical Applications}

The first application we shall consider is in the construction of functions once their singularities are known.

Example: Suppose that we wish to find an explicit expression, preferably in closed form, for the function $f(z)$ that possesses the following properties:

1. it is holomorphic everywhere except for a branch cut along the real axis segments $-\infty<x \leq-1$ and $1 \leq x<\infty$ and a simple pole of residue -1 at $z=0$;

2. it goes uniformly to zero as $|z| \rightarrow \infty$;

3. it satisfies $f^{\star}\left(z^{\star}\right)=f(z)$ and so is real on the real axis segment $-1<x<1$;

4. its discontinuity across the cut is given by $\operatorname{Im} f_{+}(x)=+\left(x^{2}+1\right)^{-1}$ for $x>1$ and $\operatorname{Im} f_{+}(x)=-\left(x^{2}+1\right)^{-1}$ for $x<-1$.

By means of an obvious generalization of (4.2.1) to deal with a cut running along two real axis segments we can ascribe to this function the representation

$$
\begin{aligned}
f(z) & =\frac{\operatorname{Res}[f(0)]}{z}+\frac{1}{\pi} \int_{-\infty}^{-1} \frac{\operatorname{Im} f_{+}(\xi)}{\xi-z} d \xi+\frac{1}{\pi} \int_{1}^{\infty} \frac{\operatorname{Im} f_{+}(\xi)}{\xi-z} d \xi \\
& =-\frac{1}{z}-\frac{1}{\pi} \int_{-\infty}^{-1} \frac{1}{\left(\xi^{2}+1\right)(\xi-z)} d \xi+\frac{1}{\pi} \int_{1}^{\infty} \frac{1}{\left(\xi^{2}+1\right)(\xi-z)} d \xi \\
& =-\frac{1}{z}+\frac{1}{\pi} \int_{1}^{\infty} \frac{1}{\left(\xi^{2}+1\right)}\left[\frac{1}{\xi+z}+\frac{1}{\xi-z}\right] d \xi .
\end{aligned}
$$

Using partial fractions, we have

$$
\int \frac{1}{\left(\xi^{2}+1\right)(\xi+z)} d \xi=\frac{1}{z^{2}+1} \ln \frac{x+z}{\sqrt{x^{2}+1}}+\frac{z}{z^{2}+1} \arctan x
$$

and

$$
\int^{x} \frac{1}{\left(\xi^{2}+1\right)(\xi-z)} d \xi=\frac{1}{z^{2}+1} \ln \frac{x-z}{\sqrt{x^{2}+1}}-\frac{z}{z^{2}+1} \arctan x
$$

Thus,

$$
f(z)=-\frac{1}{z}+\left.\frac{1}{\pi} \frac{1}{z^{2}+1} \operatorname{Ln} \frac{x^{2}-z^{2}}{x^{2}+1}\right|_{1} ^{\infty}=-\frac{1}{z}-\frac{1}{\pi} \frac{1}{z^{2}+1} \operatorname{Ln} \frac{1-z^{2}}{2} .
$$


The only limit that is placed on the diversity of applications of this sort is our capacity to conjure up unique combinations of singular behaviour.

A further application of dispersion relations involves the evaluation of principal value integrals. If we let $z$ approach the cut on the real axis from above, (4.1.5) becomes

$$
f_{+}(x)=\lim _{\delta \rightarrow 0^{+}} \frac{1}{\pi} \int_{x_{0}}^{\infty} \frac{\operatorname{Im} f_{+}(\xi)}{\xi-x-i \delta} d \xi=\frac{1}{\pi} \wp \int_{x_{0}}^{\infty} \frac{\operatorname{Im} f_{+}(\xi)}{\xi-x} d \xi+i \operatorname{Im} f_{+}(x)
$$

or,

$$
\operatorname{Re} f_{+}(x)=\frac{1}{\pi} \wp \int_{x_{0}}^{\infty} \frac{\operatorname{Im} f_{+}(\xi)}{\xi-x} d \xi, \quad x>x_{0}
$$

A similar result obtains when this limiting procedure is applied to (4.1.2) :

$$
\operatorname{Re} f_{+}(x)=\sum_{k=1}^{n} \frac{r_{k}}{x-z_{k}}+\frac{1}{\pi} \wp \int_{x_{0}}^{\infty} \frac{\operatorname{Im} f_{+}(\xi)}{\xi-x} d \xi, \quad x>x_{0} .
$$

Thus, integrals of the type $\wp \int_{c}^{\infty} \frac{g(\xi)}{\xi-x} d \xi, x>c, g(x)$ continuous on $c \leq x<\infty$, can be evaluated by identifying the harmonic conjugate of $g(x, y)$, where $g(x, 0) \equiv g(x)$. The process of identification involves intuition, guesswork, trial and error, or a combination of all three which means that the evaluation of an arbitrary integral of this type is by no means a straightforward exercise. Fortunately, it is neither difficult nor particularly time-consuming to generate a table of standard integrals to serve as an intuitive guide. The next two examples will demonstrate just how easy is this latter task.

Examples: Consider the function $f(z)=\frac{(z-1)^{\alpha-1}}{(z+1)^{\alpha}}, 0<\alpha<1$ whose behaviour at the branch points $z= \pm 1$ and at infinity permits an unsubtracted dispersion representation and hence, use of (4.3.1). If we choose the branch cut to lie along the real axis segment $-1 \leq x \leq 1$, the principal branch of this function ( the branch that is real for real $z$ not on the cut ) will be $f(z)=\frac{r_{+}^{\alpha-1}}{r^{\alpha}} e^{i\left[(\alpha-1) \theta_{+}-\alpha \theta_{-}\right]},-\pi<\theta_{ \pm}<\pi$ where $r_{ \pm}$and $\theta_{ \pm}$are defined as shown below.

Just above the cut $r_{+}=\lim _{y \rightarrow 0} \sqrt{(1-x)^{2}+y^{2}}=(1-x), r_{-}=1+x, \theta_{+}=\pi^{-}$and $\theta_{-}=0^{+}$. Therefore,

$$
f_{+}(x)=\frac{(1-x)^{\alpha-1}}{(1+x)^{\alpha}} e^{i \pi(\alpha-1)}=-\frac{(1-x)^{\alpha-1}}{(1+x)^{\alpha}} e^{i \pi \alpha} .
$$

Taking its real and imaginary parts and substituting them into (4.3.1), modified for the finite length of the cut, we obtain the simple result

$$
\wp \int_{-1}^{1} \frac{(1-\xi)^{\alpha-1}}{(1+\xi)^{\alpha}} \frac{1}{\xi-x} d \xi=\pi \cot \pi \alpha \frac{(1-x)^{\alpha-1}}{(1+x)^{\alpha}}
$$




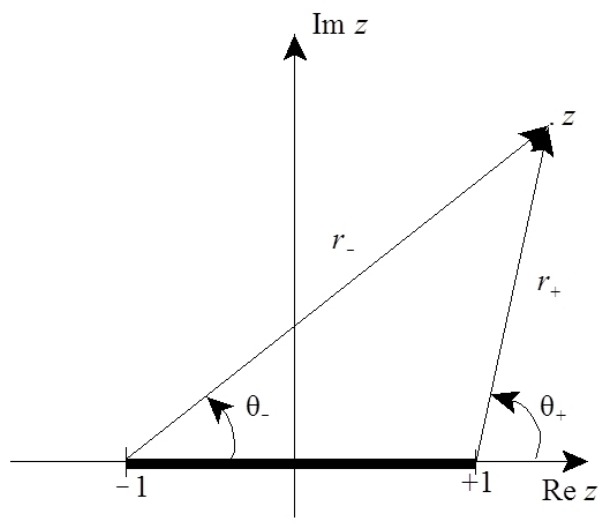

Figure 4.3: The variables used to specify the branches of $f(z)=(z-1)^{\alpha-1} /(z+1)^{\alpha}$.

As a final application, we consider the function $f(z)=\frac{\operatorname{Ln}(a-z)}{z}, a>0$ which has branch points at $z=a$ and $z=\infty$ and a simple pole at $z=0$ with residue $\operatorname{Ln} a$. In addition, with its cut chosen to lie along the positive real axis, it has the requisite behaviour to admit a dispersion representation and hence, application of (4.3.2).

With this choice of cut,

$$
\operatorname{Ln}(a-z)=\ln |a-z|+i \arg (a-z), \quad-\pi<\arg (a-z)<\pi .
$$

and so, just above the cut

$$
f_{+}(x)=\frac{1}{x} \ln |a-x|-\frac{i \pi}{x} .
$$

Therefore, (4.3.2) immediately yields

$$
\frac{1}{x} \ln |a-x|=\frac{\ln a}{x}+\frac{1}{\pi} \wp \int_{a}^{\infty} \frac{-\pi}{\xi} \frac{1}{\xi-x} d \xi, \quad x>a
$$

or,

$$
\wp \int_{a}^{\infty} \frac{1}{\xi(\xi-x)} d \xi=-\frac{1}{x} \ln \frac{x-a}{a}
$$

